\title{
Bladder Malignant Granular Cell Tumor with EP300 Gene Mutation: A Case Report and Literature Review
}

\author{
Di Zhu*, Xinle Ren*, Yi Luo, Bing Huang, Jian Huang\# \\ The Center of Pathological Diagnosis and Research, Affiliated Hospital, Guangdong Medical University, Zhanjiang, China \\ Email: \#18665763598@163.com
}

How to cite this paper: Zhu, D., Ren, X.L., Luo, Y., Huang, B. and Huang, J. (2021) Bladder Malignant Granular Cell Tumor with EP300 Gene Mutation: A Case Report and Literature Review. Journal of Cancer Therapy, 12, 240-253.

https://doi.org/10.4236/jct.2021.125023

Received: April 25, 2021

Accepted: May 11, 2021

Published: May 14, 2021

Copyright $\odot 2021$ by author(s) and Scientific Research Publishing Inc. This work is licensed under the Creative Commons Attribution International License (CC BY 4.0).

http://creativecommons.org/licenses/by/4.0/

(c) (i) Open Access

\begin{abstract}
Background: Malignant granular cell tumor (GCT) is extremely rare. Malignant GCT with EP300 gene mutation in the bladder has not been reported in the literature. Case Presentation: We report a special case of 45 -year-old female with malignant GCT of the bladder. Pathological examination showed that the mass was $11 \times 11 \times 4.5 \mathrm{~cm}$ in size, involved in the bladder's posterior wall. Under the microscope, the tumor cells were arranged in the shape of a nest or cord to infiltrate the bladder's wall. The tumor cells were pleomorphic, red-stained granular within the cytoplasm, with increased nuclear/cytoplasmic ratio, vacuolar nuclei, and obvious nucleoli. The tumor cells were showed obvious nuclear atypia, and the mitosis was more than 5/50HPF. Coagulative necrosis was widely showed within the tumor. Immunohistochemistry (IHC) showed that S-100, NSE, CD68, CR, $\alpha$-AT, and TFE-3 were strongly positive, and the Ki-67 proliferation index was around $15 \%$. The next-generation high throughput sequencing indicated that EP300 gene was missense mutated (c.457A > G) with 33\% mutation abundance, and genes of DPYD (c.1627A > G), ERCC1 (c.354T > C), NQO1 (c.559C > T), TPMT (c.719A > G) and XRCC1 (c.1196A > G) were polymorphic mutated. The patient died after three months of the second surgical treatment. Conclusions: We report for the first time a primary bladder malignant GCM accompanied by mutations in special driving genes such as EP300. We also conducted a comprehensive literature review and an in-depth discussion.
\end{abstract}

\section{Keywords}

Malignant Granular Cell Tumor, Bladder, Histopathology, EP300, Next-Generation High Throughput Sequencing 


\section{Background}

Granular cell tumor (GCT) is a benign tumor thought to originate from nerve sheath cells. It accounts for about $2 \%$ of soft tissue tumors and could occur in any part of the body [1] [2] [3] [4] [5]. The most common sites are the mouth, skin, soft tissue, breast, and female reproductive organs. GCT occurred in the bladder is rare, and only a few cases have been reported [6] [7] [8] [9]. Malignant GCT is an extremely rare soft tissue malignant tumor, accounting for less than $2 \%$ of all soft tissue GCTs. Malignant GCT occurs in a wide range of ages, mostly in adults aged 30 - 70, with an average age of 50. Most of the clinical manifestations are painless masses in subcutaneous or deep soft tissue. Most of the cases occurred in the extremities, torso, head, neck, and breast [10]-[16]. To date, few cases of bladder malignant GCT have been reported [7] [17]. Few studies on the driving genes of malignant GCT, especially the specific driving genes of malignant GCT, are not clear [1] [18] [19]. Here, we report a case of malignant bladder GCT with vulvar, vaginal, and pelvic soft tissue invasion. Histopathology and IHC confirmed that it was a malignant GCT originating from the bladder. The next-generation high throughput sequencing detected several gene mutations, including a tumor-specific driving gene EP300 and another five genes associated with chemotherapy. We also conducted a literature review and in-depth discussion.

\section{Case Presentation}

The patient was a 45-year-old Chinese female, housewife and Han nationality. The patient found pelvic mass during physical examination a year ago, the clinical manifestations were intermittent lower abdominal discomfort, frequent urination, dysuria, no hematuria, no vaginal bleeding. The outpatient clinic was admitted to the hospital with "pelvic mass". The patient was without any treatment before. B-ultrasound examination showed that the left side of the uterus was abnormally hypoechoic $(10.4 \times 9.6 \mathrm{~cm})$, the right ovary had no obvious abnormality, and the left ovary showed unclearly. Then she was admitted to the hospital as a "pelvic mass". The patient was treated with abdominal hysterectomy and double salpingectomy, pelvic tumor resection, cystectomy, and ureterostomy under general anesthesia.

Magnetic resonance imaging (MRI) pelvic plain scanning showed a large lobulated mass among the cervix, the anterior wall of the vagina, and below the bladder. The mass was $12 \times 11 \times 10 \mathrm{~cm}$ in size with clear boundary. T1W1 showed a slightly higher signal, T2W1 showed a high-low mixed-signal, and DWI showed a mixed high signal. An enhanced scan showed obvious uneven enhancement, uterine body, and cervical lifting. The bladder was connected tightly to the mass and compressed forward and upward (Figure 1). The mass in the pelvis was closely adhesive to the base of the bladder and the wall of the uterus. After complete hysterectomy, a huge mass was found between the anterior wall of the vagina and the posterior wall of the bladder. The anterior boundary of the 
tumor was not separated from the posterior wall of the bladder and protruded into the bladder. When the bladder was completely dissociated from the extraperitoneal cavity, it was seen that the tumor originated from the posterior wall of the bladder and infiltrate to the anterior wall of the urethra to the pelvic floor.

Total cystectomy was performed, and the tumor with size of $11 \times 11 \times 4.5 \mathrm{~cm}$ was detected at the posterior wall of the bladder. The section of the tumor was grayish-yellow, tough, and lobulated. The tumor involved the whole layer of the bladder wall, did not break through the bladder adventitia, and the boundary between the tumor and the surrounding tissue was still clear (Figure 2).
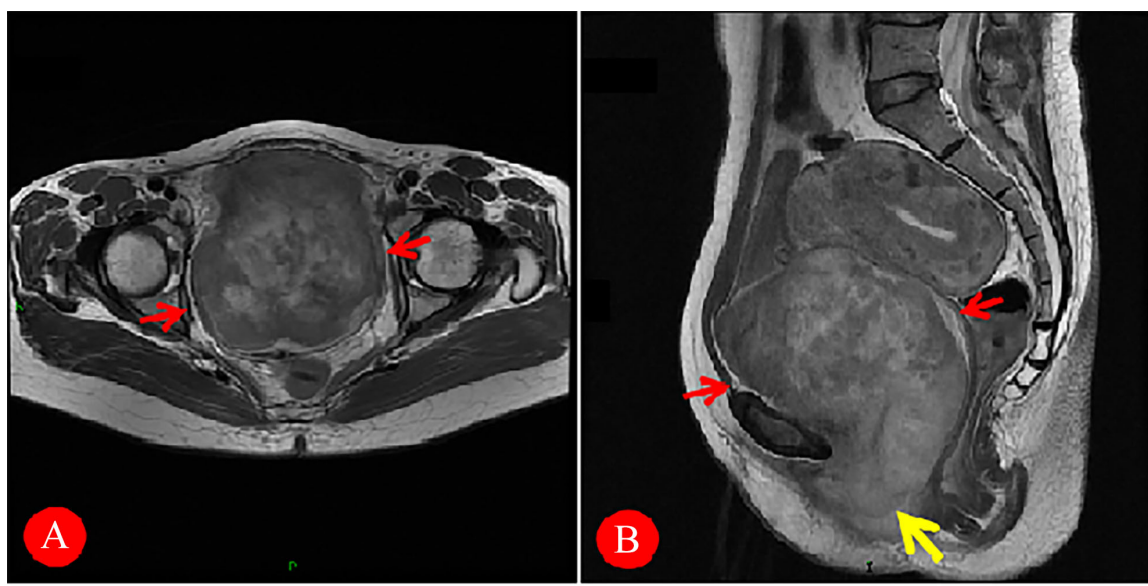

Figure 1. MRI showed a large mass between the cervix, the anterior wall of the vagina, and the bottom of the bladder, and the boundary was still clear. (A) A coronal section (The red arrow showed the extent of the tumor); (B) A sagittal section (the red arrow showed the extent of the tumor, the yellow arrow showed the connection between the tumor and the bottom of the bladder.).
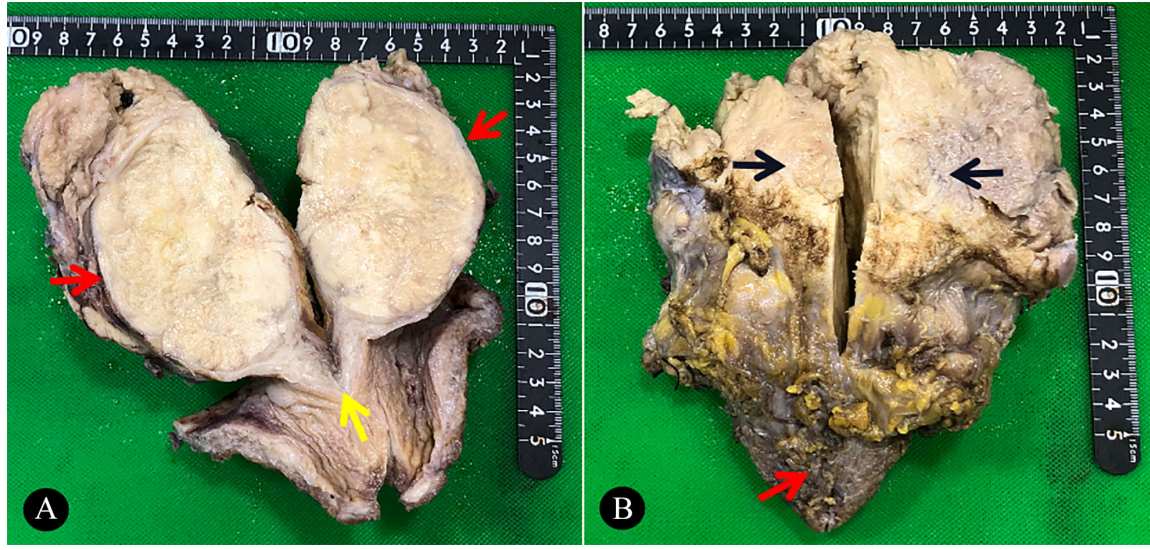

Figure 2. A volume of $11 \times 11 \times 4.5 \mathrm{~cm}$ in tumor size was collected to the posterior wall of the bladder. (A) The section of the tumor was grayish-yellow, tough, and lobulated. The red arrow showed the extent of the tumor, the yellow arrow showed the connection between the tumor and the bottom of the bladder. (B) Back view: The black arrow shows the adhesion between the tumor and the pelvic soft tissue. The red arrow showed the bottom of the bladder. 
After surgical removal, hematoxylin and eosin (H\&E) staining were performed on tumor tissue. The tumor tissue was separated by fibrous connective tissue, arranged in flake, nest and cord shape, showing infiltrative growth. The tumor cells were pleomorphic, round, polygonal, and fusiform. The ratio of the nucleus to the cytoplasm was increased. The cytoplasm was rich in red staining with a large number of eosinophilic bodies. The nuclei were heteromorphic with different sizes. Some of the nuclei were vacuolated and had obvious nucleoli. Multinucleated tumor cells and scattered coagulative necrotic foci were seen locally, and the mitotic figure was more than 5/50HPF (Figure 3).

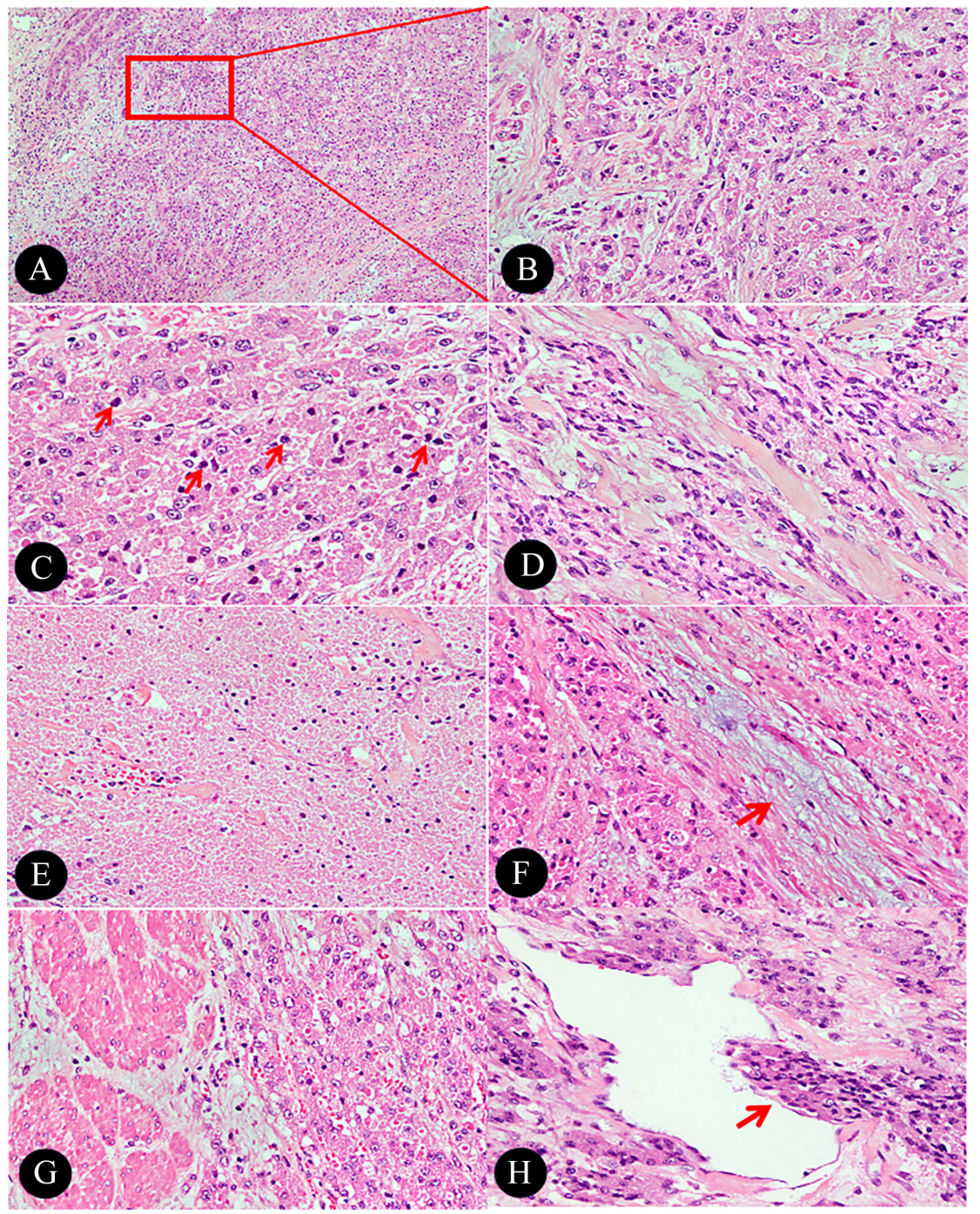

Figure 3. Under a microscope: (A) Overall the histopathology. (B) The tumor cells were pleomorphic, round, polygonal, and fusiform. The ratio of the nucleus to the cytoplasm was increased. The cytoplasm was rich in red staining with a large number of eosinophilic bodies. (C) The mitotic figure was more than 5/50HPF (shown by the red arrow). (D) The tumor tissue was separated by fibrous connective tissue, arranged in flake, nest and cord shape. (E) Coagulative necrosis. (F) Interstitial myxoid degeneration shown by the red arrow. (G) The tumor cells invaded the bladder muscle wall. $(\mathrm{H})$ The tumor cells invade the lumen of blood vessels (shown by the red arrow). 
DAKO company's latest Omnis automatic immunohistochemical instrument was used for staining, and the process was carried out under the operation manual provided by the manufacturer. The sources, clones, and manufacturer of the antibodies used are shown in Table 1 . Negative and positive controls were all set up for each antibody. The IHC results showed that S-100, NSE, CD68, CR, $\alpha$-AT, and TFE-3 in the tumor cells were strongly positive, and the Ki-67 proliferation index in most of the tumor areas was around 15\% (Figure 4). The IHC results showed that SMA, Desmin, $\alpha$-inhibin, GFAP, MyoD1, HMB45, and CK (pan) were all negative (not shown).

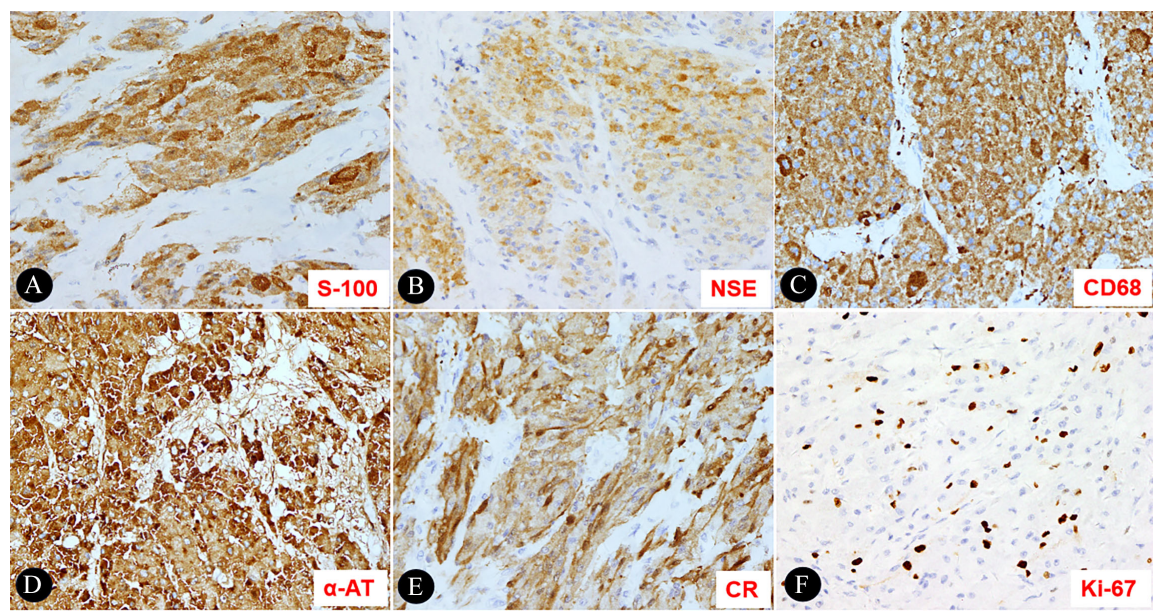

Figure 4. The IHC results showed that S-100 (A), NSE (B), CD68 (C), $\alpha$-AT (D), CR (E), and TFE- 3 in the tumor cells were strongly positive, and Ki-67 proliferation index in most of the tumor areas was around $15 \%(\mathrm{~F})$.

Table 1. The sources, clones and manufacturer of the antibodies used in this study.

\begin{tabular}{ccc}
\hline Antibodies & Clones & Manufacturers \\
\hline CD68 & KP1 & MXB \\
NSE & $3-3-C$ & MXB \\
S-100 & 4 C4.9 & MXB \\
CR(Calretinin) & MX027 & MXB \\
$\boldsymbol{\alpha}$-AT(AAT) & Polyclone & MXB \\
TFE-3 & MRQ-37 & MXB \\
SMA & 1 A4 & MXB \\
Desmin & MX046 & MXB \\
$\boldsymbol{\alpha}$-inhibin & AMY82 & MXB \\
GFAP & MX047 & MXB \\
Ki-67 & MIB-1 & Dako \\
MyoD1 & $5.8 \mathrm{~A}$ & MXB \\
HMB45 & HMB45 & MXB \\
CK(pan) & AE1/AE3 & Dako \\
& &
\end{tabular}


Next-generation high-throughput sequencing (NGS) covered 425 gene exons, fusion-related introns, variable splicing regions, specific microsatellite (MS) sites, and other common $1.46 \mathrm{Mb}$ base sites were used in this study. The whole sequencing process was performed on the Illumina-based Hiseq 4000 Sequencing system at Nanjing Geneseeq Technology Inc., Nanjing, Jiangsu, China. In the sequencing of the 425 genes, the results of NGS indicated that the overall mutation rate is very low. The results revealed that the EP300 gene was missense mutated (c.457A > G) with 33\% mutation abundance. The gene of EP300 is in the classification of "key genes in the tumor-related signaling pathways". The NGS results also showed that genes of DPYD (c.1627A > G), ERCC1 (c.354T > C), NQO1 (c.559C > T), TPMT (c.719A > G), and XRCC1 (c.1196A > G) were polymorphic mutated. These genes are all in the classification of "key genes for chemotherapy". The detailed locations and functions are shown in Table 2. NGS results also showed that the tumor mutational burden (TMB) was very lower (2.1 mutations/Mb), and the microsatellite (MS) analysis showed that no MIS-H related genes were detected. The list of the 425 genes and functional partition was shown in supplement data (Table 3).

Three months after the first operation, the patient was re-admitted to the hospital because of "vaginal mass found for more than one week". Specialist examination: a $3 \mathrm{~cm}$ lobulated moveable tenderness, clear boundary mass could be touched the right vaginal wall, and the mass was close to the orifice of the vulva. Surgical findings: the vulvar mass was located in the lower part of the vagina behind the right greater labia. It was a lobulated hard tumor, with a size of about 4 $\times 3 \times 1 \mathrm{~cm}$ and clear boundary. The patient died three months after the second operation.

Table 2. The mulations of gens detected by NGS.

\begin{tabular}{|c|c|c|c|c|}
\hline Genes & Variations & Mutant types & Ways of mutation & Possible functions \\
\hline EP300 & p.M153V & c.457A > G(p.M153V) & $\begin{array}{l}\text { Missense mutation } \\
\text { in exon } 2 \\
\left(33 \% \text { in } \mathrm{MF}^{*}\right)\end{array}$ & Possible tumor driving genes \\
\hline DPYD & p.I153V & c.1627A > G(p.I153V) & $\begin{array}{l}\text { Heterozygous } \\
\text { polymorphism }\end{array}$ & $\begin{array}{c}\text { Side effects may increase for } \\
\text { drugs of 5-FU, capecitabine, etc. }\end{array}$ \\
\hline ERCC1 & p.N118N & c.354T > C(p.N118=) & $\begin{array}{l}\text { Heterozygous } \\
\text { polymorphism }\end{array}$ & $\begin{array}{l}\text { The curative effect by using } \\
\text { drugs of platinum may be } \\
\text { better than that of the wild type. }\end{array}$ \\
\hline NQO1 & p.P187S & c.559C > T(p.P187S) & $\begin{array}{l}\text { Heterozygous } \\
\text { polymorphism }\end{array}$ & $\begin{array}{l}\text { The curative effect by using } \\
\text { mitomycin } C \text { may be worse } \\
\text { than that of the wild type. }\end{array}$ \\
\hline TPMT & p.Y240C & c.719A > G(p.Y240C) & $\begin{array}{l}\text { Heterozygous } \\
\text { polymorphism }\end{array}$ & $\begin{array}{l}\text { Toxic side effects by using } \\
\text { mercaptopurine may increase }\end{array}$ \\
\hline XRCC1 & p.Q399R & c.1196A > G(p.Q399R) & $\begin{array}{l}\text { Heterozygous } \\
\text { polymorphism }\end{array}$ & $\begin{array}{l}\text { The curative effect by using } \\
\text { drugs of platinum may be } \\
\text { better than that of the wild type. }\end{array}$ \\
\hline
\end{tabular}

${ }^{\star}$ Mutation Frequency. 
Table 3. Supplement data.

\begin{tabular}{|c|c|c|c|c|c|c|}
\hline ABCB1(MDR1) & CDK12 & ERCC2 & IDH1 & MSH6 & PPARD & SMO \\
\hline ABCC2(MRP2) & CDK4 & ERCC3 & IDH2 & MTHFR & PPP2R1A & SOCS1 \\
\hline $\mathrm{ADH} 1 \mathrm{~B}$ & CDK6 & ERCC4 & IFNA6 & MTOR & PRDM1 & SOS1 \\
\hline AIP & CDK8 & ERCC5 & IFNB1 & MUTYH & PREX2 & SOX2 \\
\hline AKT1 & CDKN1A & ESR1 & IFNE & MYC & PRF1 & SPOP \\
\hline AKT2 & CDKN1B & ETV1 & IFNG & MYCL & PRKACA & SPRED1 \\
\hline AKT3 & CDKN1C & ETV4 & IFNGR1 & MYCN & PRKAR1A & SPRY4 \\
\hline ALDH2 & CDKN2A & ETV5 & IFNGR2 & MYD88 & PRKCI & SRC \\
\hline ALK & CDKN2B & ETV6 & IGF1R & MYH9 & PRKDC & SRSF2 \\
\hline AMER1 & CDKN2C & EWSR1 & IGF2 & NAT1 & PRSS1 & SRY \\
\hline APC & CEBPA & EXT1 & IKBKE & NBN & PRSS3 & STAG2 \\
\hline $\mathrm{AR}$ & CEP57 & EXT2 & IKZF1 & NCOR1 & PTCH1 & STAT3 \\
\hline ARAF & CHD4 & $\mathrm{EZH} 2$ & IL7R & NF1 & PTEN & STK11 \\
\hline ARID1A & CHD8 & EZR & INPP4B & NF2 & PTK2 & STMN1 \\
\hline ARID1B & CHEK1 & FANCA & IRF2 & NFE2L2 & PTPN11 & SUFU \\
\hline ARID2 & CHEK2 & FANCC & JAK1 & NFKBIA & PTPN13 & TACC3 \\
\hline ARID5B & CREBBP & FANCD2 & JAK2 & NKX2-1 & QKI & TAP1 \\
\hline ASCL4 & CRKL & FANCE & JAK3 & NOTCH1 & $\mathrm{RAC1}$ & TAP2 \\
\hline ASXL1 & CSF1R & FANCF & JARID2 & NOTCH2 & RAC3 & TEK \\
\hline ATF1 & CTCF & FANCG & JUN & NOTCH3 & RAD50 & TEKT4 \\
\hline ATIC & CTLA4 & FANCI & KDM5A & NPM1 & RAD51 & TERC \\
\hline ATM & CTNNB1 & FANCL & KDR(VEGFR2) & NQO1 & RAD51B & TERT \\
\hline ATR & CUL3 & FANCM & KEAP1 & NRAS & RAD51C & TET2 \\
\hline ATRX & CUX1 & FAT1 & KIF1B & NRG1 & RAD51D & TGFBR2 \\
\hline AURKA & CXCR4 & FBXW7 & KIT & NSD1 & RAD54L & THADA \\
\hline AURKB & CYLD & FGF19 & KITLG & NTRK1 & RAF1 & TMEM127 \\
\hline AXIN2 & CYP19A1 & FGFR1 & KLLN & NTRK2 & RARA & TMPRSS2 \\
\hline AXL & CYP2A13 & FGFR2 & KMT2A(MLL) & NTRK3 & RARG & TNFAIP3 \\
\hline B2M & CYP2A6 & FGFR3 & KMT2B & NUTM1 & RASGEF1A & TNFRSF11A \\
\hline BAD & CYP2A7 & FGFR4 & KMT2C & PAK3 & RB1 & TNFRSF14 \\
\hline BAI3 & CYP2B6*6 & FH & KMT2D(MLL2) & PALB2 & RECQL4 & TNFRSF19 \\
\hline BAK1 & CYP2C19*2 & FLCN & KRAS & PALLD & RELN & TNFSF11 \\
\hline BAP1 & $\mathrm{CYP} 2 \mathrm{C} 9 * 3$ & FLT1(VEGFR1) & LHCGR & PARK2 & RET & TOP1 \\
\hline
\end{tabular}


Continued

\begin{tabular}{|c|c|c|c|c|c|c|}
\hline BARD1 & CYP2D6 & FLT3 & LMO1 & PARP1 & RHOA & TOP2A \\
\hline BAX & $\mathrm{CYP} 3 \mathrm{~A} 4^{\star} 4$ & FLT4 & LRP1B & PARP2 & RICTOR & TP53 \\
\hline BCL2 & CYP3A5 & FOXA1 & LYN & PAX5 & RNF43 & TP63 \\
\hline BCL2L11(BIM) & CYSLTR2 & FOXL2 & LZTR1 & PBRM1 & ROS1 & TPMT \\
\hline BCR & DAXX & FOXP1 & MAP2K1(MEK1) & PDCD1(PD1) & RPTOR & TSC1 \\
\hline BIRC3 & DDR2 & FRG1 & MAP2K2(MEK2) & PDCD1LG2(PD-L2) & RRM1 & TSC2 \\
\hline BLM & DENND1A & GATA1 & MAP2K4 & PDE11A & RUNX1 & TSHR \\
\hline BMPR1A & DHFR & GATA2 & MAP3K1 & PDGFRA & RUNX1T1 & TTF1 \\
\hline BRAF & DICER1 & GATA3 & MAP3K4 & PDGFRB & SBDS & TUBB3 \\
\hline BRCA1 & DLL3 & GATA4 & MAX & PDK1 & SDC4 & TYMS \\
\hline BRCA2 & DNMT3A & GATA6 & MCL1 & PGR & SDHA & U2AF1 \\
\hline BRD4 & DOT1L & GNA11 & MDM2 & PHOX2B & SDHB & UGT1A1 \\
\hline BRIP1 & DPYD & GNAQ & MDM4 & PIK3C3 & SDHC & VAMP2 \\
\hline BTG2 & DTL(CDT2) & GNAS & MECOM & PIK3CA & SDHD & VEGFA \\
\hline BTK & DUSP2 & GRIN2A & MED12 & PIK3CD & 9-Sep & VHL \\
\hline BUB1B & EGFR & GRM3 & MEF2B & PIK3R1 & SETBP1 & WAS \\
\hline c11orf30 & EIF1AX & GRM8 & MEN1 & PIK3R2 & SETD2 & WISP3 \\
\hline CASP8 & EP300 & GSTM1 & MET & PKHD1 & SF3B1 & WRN \\
\hline CBL & EPAS1 & GSTM4 & MGMT & PLAG1 & SGK1 & WT1 \\
\hline CBLB & EPCAM & GSTP1 & MITF & PLCB4 & SKP2 & XPA \\
\hline CCND1 & EPHA2 & GSTT1 & MLH1 & PLK1 & SLC34A2 & XPC \\
\hline CCNE1 & EPHA3 & HDAC2 & MLH3 & PMS1 & SLC3A2 & XRCC1 \\
\hline CD274(PD-L1) & EPHA5 & HDAC9 & MLLT1 & PMS2 & SMAD2 & XRCC2 \\
\hline CD74 & ERBB2(HER2) & HGF & MLLT3 & POLD1 & SMAD3 & YAP1 \\
\hline CDA & ERBB2IP & HLA-A & MLLT4 & POLD3 & SMAD4 & ZNF217 \\
\hline CDC73 & ERBB3 & HNF1A & MPL & POLE & SMAD7 & ZNF703 \\
\hline CDH1 & ERBB4 & HNF1B & MRE11A & POLH & SMARCA4 & \\
\hline CDK10 & ERCC1 & HRAS & MSH2 & POT1 & SMARCB1 & \\
\hline & \multicolumn{6}{|c|}{ Key genes for targeted drug use } \\
\hline & \multicolumn{6}{|c|}{ Key genes for chemotherapy } \\
\hline & \multicolumn{6}{|c|}{ Genetic risk prediction genes } \\
\hline & \multicolumn{6}{|c|}{ Important tumor-driving genes, but there are no targeted drugs at present } \\
\hline & enes in $t$ & $d$ & & & & \\
\hline
\end{tabular}




\section{Discussion and Conclusions}

The diagnostic criteria for malignant GCT were proposed by Fanburg-Smith and his colleagues as early as 1998. Malignant histological criteria included coagulative necrosis, fusiform, vesicular nucleus of large nucleolus, increased mitotic activity ( $>2$ mitoses/high magnification $\times 200$ ), increased nuclear/cytoplasmic ratio, and pleomorphism [20]. According to the pathological criteria for the diagnosis of malignant GCT, both histopathology and immunohistochemical expression of this presented case support the diagnosis of malignant GCT [20]. In addition to the criteria of histopathology, in this case, we also identified biological changes associated with malignant characteristics, such as invasive growth and vascular invasion. The differential diagnosis of benign and malignant GCT is often tricky in both pathology and clinic. However, this case is not difficult, based on histopathology and biological behavior. The tumor originated from the bladder wall and invaded the muscular layer of the bladder. In addition, the tumor also widely invaded into vagina, cervix, and soft tissues in the pelvic, showing extremely malignant biological behavior. The patient did not respond to chemotherapy and died three months after the second operation.

Malignant GCM is extremely rare, and few cases have been reported [7] [11] [17] [21] [22]. So far, we know little about the molecular biology and molecular genetics of malignant GCT. Recently, Richard Davis et al. reported a case of malignant GCT of the lung with ATM gene mutation. ATM gene encodes DNA repair protein involved in cell cycle regulation [1]. Xu and colleagues apply the NGS to identify mutations in the genes of ASXL, NOTCH, and PARP pathways as potential driver mutations in pulmonary malignant GCT [23].

In our case, based on the high-throughput second-generation sequencing of 425 genes, the overall mutation rate of the tumor was very low. In the classification of "the key genes in tumor-related signaling pathway", the EP300 gene was missense mutated (C.457A > G) with 33\% mutation abundance. As we know, EP300 gene is located on chromosome 22, and encodes adenovirus E1A-related intracellular p300 protein, which has acetyltransferase activity, regulates transcriptional activity through chromosome remodeling. EP300 and its homolog CREB binding protein (CBP) are two lysine acetyltransferases, which act as transcriptional factors and play an essential role in developing, progression, and treating many diseases, especially in tumors. Some cancer-related point mutations are gain-of-function alterations in EP300/CBP that might also contribute to cancer development [24] [25] [26]. It has been found that the inactivation mutations of histone acetyltransferase CBP and EP300 are prevalent in diffuse large B cell lymphoma (DLBCL). The functional inactivation mutations of CBP and EP300 will down-regulate the expression of $\mathrm{CD} 20$, which leads to the failure of anti-CD20 antibody therapy [27]. Other studies have also shown that targeting EP300 may effectively treat fatal leukemia [28]. Therefore, the use of synthetic small-molecular compounds to inhibit the function of mutant EP300 brings hope for targeted therapy of malignant tumors [29]. A recent study also shows that the 
EP300 protein is related to the grade, pathological T stage, lymph node metastasis, and survival rate of esophageal squamous cell carcinoma [30].

Gongmin Zhu and colleagues found that EP300 mutation in bladder cancer is associated with a higher tumor mutation burden (TMB) and up-regulates the immune system's signal pathway, which can be used as a biomarker to predict the effect of immunotherapy [31]. In our case, NGS results showed that the TMB was significantly lower (2.1 mutations/Mb). The relationship between EP300 mutation and TMB may be related to different tumor types and mutation sites. How genetic or epigenetic changes in EP300 will affect the phenotype of various cancers is far from clear.

In our case, in addition to the EP300 missense mutation, it was also detected that DPYD, NQO1 and TPMT had heterozygous polymorphism mutations; ERCC1 and XRCC1 had homozygous polymorphism mutations. These genes are all in the classified as "the key genes for chemotherapy" in total 425 genes. Heterozygous mutation of the DPYD gene in p. I543V is a missense mutation caused by single nucleotide polymorphism rs1801159, which can decrease the activity of DPYD, increase the toxicity and side effects of 5-fluorouracil, and increase the risk of vomiting and nausea in patients [32]. ERCC1 gene p.N118N homozygous mutation is a synonymous mutation caused by single nucleotide polymorphism rs11615. This homozygous genotype can increase the risk of lung cancer and breast cancer. Besides, this mutation can reduce mRNA level by affecting the stability of ERCC1 transcripts and increase cell response to platinum drugs by reducing DNA repair function [33]. TPMT gene p.Y240C heterozygous mutation $\left(\mathrm{TPMT}^{\star} 3 \mathrm{C}\right.$ ) is a missense mutation caused by single nucleotide polymorphism rs1142345. This heterozygous polymorphism mutation can reduce mercaptopurine methyltransferase activity by about $50 \%$, which leads to serious poor metabolism of mercaptopurine and side effects [34]. XRCC1 gene p.Q399R homozygous mutation is a missense mutation caused by single nucleotide polymorphism rs25487, which is associated with the risk of non-small cell lung cancer, breast cancer, colorectal cancer, gastric cancer, and other tumors. This homozygous mutation is related to platinum chemotherapeutic drugs' efficacy and can increase the response rate of cells to platinum drugs [35].

There is no targeted drug for malignant GCT, and whether it is sensitive to chemotherapy may be related to its particular gene mutation. Wei, L, and colleagues performed whole-genome sequencing of a malignant GCT that was metabolically responsive to pazopanib. The results showed that the mutation rate was shallow, and the whole genome was stable [19]. Due to the small number of cases, there are not many reports on the survival of malignant GCM. However, it is generally believed that prognosis of GCM is closely related to the tumor's size, local invasion, and distant metastasis [22] [36]. It took less than a year from discovery to death in our case.

In summary, few cases of malignant GCM of the bladder have been reported, but none of them have been studied by molecular genetics. We performed mo- 
lecular genetic analysis of malignant GCM of the bladder for the first time, and in particular, detected a missense mutation in exon 2 of EP300 gene p.M153V with a high mutation abundance. The clinical significance of EP300 gene mutation in malignant GCM of the bladder is not clear. Whether it leads to the EP300 protein dysfunction to activate downstream signal pathways and participates in tumorigenesis and development are not certified. More clinical cases and experimental studies are needed for further exploration.

\section{Declarations}

\section{Ethics Approval and Consent to Participate}

This case was reviewed and approved by the Ethics Committee of Affiliated Hospital, Guangdong Medical University, Zhanjiang, China.

\section{Consent for Publication}

Written informed consent was obtained from the patient's next of kin for publication of this case report and any accompanying images. A copy of the written consent is available for review by the Editor-in-Chief of this journal.

\section{Availability of Data and Materials}

All data generated and described in this article are freely available to any scientist wishing to use them for noncommercial purposes, without breaching participant confidentiality.

\section{Authors' Contributions}

J.H. found the specific case; J.H. and B.H., two senior diagnostic pathologist made the final pathological diagnosis for the case; J.H. and D.Z. wrote the main manuscript text; D.Z., XL.R., Y.L. and J.H. prepared Figures 1-4; All authors reviewed the manuscript.

\section{Conflicts of Interest}

The authors declare no conflicts of interest regarding the publication of this paper.

\section{References}

[1] Davis, R., Deak, K. and Glass, C.H. (2019) Pulmonary Granular Cell Tumors: A Study of 4 Cases Including a Malignant Phenotype. The American Journal of Surgical Pathology, 43, 1397-1402. https://doi.org/10.1097/PAS.0000000000001303

[2] Stemm, M., et al. (2017) Typical and Atypical Granular Cell Tumors of Soft Tissue: A Clinicopathologic Study of 50 Patients. American Journal of Clinical Pathology, 148, 161-166. https://doi.org/10.1093/ajcp/aqx058

[3] Rubino, F., et al. (2020) Granular Cell Tumors of the Sellar Region: What Should Be Done after Subtotal Resection? A Systematic Review. Pituitary, 23, 721-732. https://doi.org/10.1007/s11102-020-01068-6

[4] Aoyama, K., et al. (2012) Granular Cell Tumors: A Report of Six Cases. World Journal 
of Surgical Oncology, 10, Article No. 204. https://doi.org/10.1186/1477-7819-10-204

[5] Shi, Y., et al. (2020) Experience with Esophageal Granular Cell Tumors: Clinical and Endoscopic Analysis of 22 Cases. Digestive Diseases and Sciences, 66, 1233-1239. https://doi.org/10.1007/s10620-020-06337-9

[6] Lee, C., et al. (2018) Clinicopathologic Features of Benign Neurogenic Tumor of Urinary Bladder. International Journal of Surgical Pathology, 26, 221-228. https://doi.org/10.1177/1066896917742722

[7] Sun, Y., et al. (2018) Granular Cell Tumor of the Bladder: A Report of Six Cases. Urology, 121, 203.e1-203.e5. https://doi.org/10.1016/j.urology.2018.08.018

[8] Yoshida, T., et al. (2001) Granular Cell Tumor of the Urinary Bladder. International Journal of Urology, 8, 29-31. https://doi.org/10.1046/j.1442-2042.2001.00241.x

[9] Eandi, J.A., et al. (2007) Granular Cell Tumor of the Urinary Bladder with Pseudoepitheliomatous Hyperplasia and Colocalization with Adenocarcinoma. International Journal of Urology, 14, 862-864. https://doi.org/10.1111/j.1442-2042.2007.01827.x

[10] Shah, K., et al. (2020) Malignant Granular Cell Tumor of the Lower Extremity in an Adolescent Male. Journal of Pediatric Hematology/Oncology, 43, e529-e531. https://doi.org/10.1097/MPH.0000000000001886

[11] Stasek, M., et al. (2020) Malignant Granular Cell Tumor of the Esophagus: A Case Report. Annals of Thoracic and Cardiovascular Surgery, 26, 359-364. https://doi.org/10.5761/atcs.cr.20-00117

[12] Bradford, B.E., et al. (2020) Benign and Malignant Granular Cell Tumor of the Hypopharynx: Two Faces of a Rare Entity. Head and Neck Pathology, 15, 281-287. https://doi.org/10.1007/s12105-020-01157-9

[13] Tsukamoto, S., et al. (2019) Malignant Granular Cell Tumor of the Median Nerve: A Case Report with a Literature Review of 157 Cases. Skeletal Radiology, 48, 307-316. https://doi.org/10.1007/s00256-018-3017-2

[14] Jia, X., et al. (2017) Large Malignant Granular Cell Tumor with Suprascapular Nerve and Brachial Plexus Invasion: A Case Report and Literature Review. Medicine (Baltimore), 96, e8531. https://doi.org/10.1097/MD.0000000000008531

[15] Akahane, K., et al. (2015) Malignant Granular Cell Tumor of the Breast: Case Report and Literature Review. Breast Cancer, 22, 317-323. https://doi.org/10.1007/s12282-012-0362-1

[16] Liu, T.T., et al. (2015) Primary Cutaneous Malignant Granular Cell Tumor: A Case Report in China and Review of the Literature. Diagnostic Pathology, 10, Article No. 113. https://doi.org/10.1186/s13000-015-0357-2

[17] Zhuang, Y.Z., Jiang, X.Y. and Chen, P.Q. (2006) Malignant Granular Cell Tumor of the Urinary Bladder. Chinese Journal of Pathology, 35, 188.

[18] Pareja, F., et al. (2018) Loss-of-Function Mutations in ATP6AP1 and ATP6AP2 in Granular Cell Tumors. Nature Communications, 9, Article No. 3533. https://doi.org/10.1038/s41467-018-05886-y

[19] Wei, L., et al. (2015) Whole-Genome Sequencing of a Malignant Granular Cell Tumor with Metabolic Response to Pazopanib. Cold Spring Harbor Molecular Case Studies, 1, a000380. https://doi.org/10.1101/mcs.a000380

[20] Fanburg-Smith, J.C., et al. (1998) Malignant Granular Cell Tumor of Soft Tissue: Diagnostic Criteria and Clinicopathologic Correlation. The American Journal of Surgical Pathology, 22, 779-794. https://doi.org/10.1097/00000478-199807000-00001

[21] Quinn, P.L., et al. (2019) Malignant Granular Cell Tumor of the Bile Duct. ACG 
Case Reports Journal, 6, e00193. https://doi.org/10.14309/crj.0000000000000193

[22] Moten, A.S., et al. (2018) Malignant Granular Cell Tumor: Clinical Features and Long-Term Survival. Journal of Surgical Oncology, 118, 891-897.

https://doi.org/10.1002/jso.25227

[23] Xu, S., et al. (2015) Next Generation Sequencing Uncovers Potential Genetic Driver Mutations of Malignant Pulmonary Granular Cell Tumor. Journal of Thoracic Oncology, 10, e106-e109. https://doi.org/10.1097/JTO.0000000000000640

[24] Attar, N. and Kurdistani, S.K. (2017) Exploitation of EP300 and CREBBP Lysine Acetyltransferases by Cancer. Cold Spring Harbor Perspectives in Medicine, 7, a026534. https://doi.org/10.1101/cshperspect.a026534

[25] Breen, M.E. and Mapp, A.K. (2018) Modulating the Masters: Chemical Tools to Dissect CBP and p300 Function. Current Opinion in Chemical Biology, 45, 195-203. https://doi.org/10.1016/j.cbpa.2018.06.005

[26] Blobel, G.A. (2000) CREB-Binding Protein and p300: Molecular Integrators of Hematopoietic Transcription. Blood, 95, 745-755.

https://doi.org/10.1182/blood.V95.3.745.003k05 $745 \quad 755$

[27] Scialdone, A., et al. (2019) Depletion of the Transcriptional Coactivators CREBBinding Protein or EP300 Downregulates CD20 in Diffuse Large B-Cell Lymphoma Cells and Impairs the Cytotoxic Effects of Anti-CD20 Antibodies. Experimental Hematology, 79, 35-46.e1. https://doi.org/10.1016/j.exphem.2019.10.004

[28] Huang, Y., et al. (2019) The Leukemogenic TCF3-HLF Complex Rewires Enhancers Driving Cellular Identity and Self-Renewal Conferring EP300 Vulnerability. Cancer Cell, 36, 630-644.e9. https://doi.org/10.1016/j.ccell.2019.10.004

[29] Yang, Y., et al. (2020) Discovery of Highly Potent, Selective, and Orally Efficacious p300/CBP Histone Acetyltransferases Inhibitors. Journal of Medicinal Chemistry, 63, 1337-1360. https://doi.org/10.1021/acs.jmedchem.9b01721

[30] Bi, Y., et al. (2019) EP300 as an Oncogene Correlates with Poor Prognosis in Esophageal Squamous Carcinoma. Journal of Cancer, 10, 5413-5426. https://doi.org/10.7150/jca.34261

[31] Zhu, G., et al. (2020) EP300 Mutation Is Associated with Tumor Mutation Burden and Promotes Antitumor Immunity in Bladder Cancer Patients. Aging (Albany NY), 12, 2132-2141. https://doi.org/10.18632/aging.102728

[32] Zhang, H., et al. (2007) DPYD*5 Gene Mutation Contributes to the Reduced DPYD Enzyme Activity and Chemotherapeutic Toxicity of 5-FU: Results from Genotyping Study on 75 Gastric Carcinoma and Colon Carcinoma Patients. Medical Oncology, 24, 251-258. https://doi.org/10.1007/BF02698048

[33] Nebert, D.W., et al. (2002) NAD(P)H: Quinone Oxidoreductase (NQO1) Polymorphism, Exposure to Benzene, and Predisposition to Disease: A HuGE Review. Genetics in Medicine, 4, 62-70. https://doi.org/10.1097/00125817-200203000-00003

[34] Almoguera, B., et al. (2014) Imputation of TPMT Defective Alleles for the Identification of Patients with High-Risk Phenotypes. Frontiers in Genetics, 5, 96. https://doi.org/10.3389/fgene.2014.00096

[35] Li, D., et al. (2007) Effects of Base Excision Repair Gene Polymorphisms on Pancreatic Cancer Survival. International Journal of Cancer, 120, 1748-1754. https://doi.org/10.1002/ijc.22301

[36] Alnashwan, Y.A., Ali, K. and Amr, S.S. (2019) Metastasizing Malignant Granular Cell Tumor (Abrikossoff Tumor) of the Anterior Abdominal Wall, with Prolonged Survival. Case Reports in Pathology, 2019, Article ID: 9576487. https://doi.org/10.1155/2019/9576487 


\section{Abbreviations}

GCT: Granular cell tumor

NGS: Next-generation high throughput sequencing

MRI: Magnetic resonance imaging

IHC: Immunohistochemistry

$\mathrm{H} \& \mathrm{E}$ : Hematoxylin and Eosin

MS: Microsatellite

TMB: Tumor Mutational Burden

CBP: CREB binding protein

DLBCL: Diffuse large B cell lymphoma 